\title{
Preparation for the Clinical Viva
}

\section{Examination format}

You need to be aware of the format of the vivas.

The day consists of two viva sessions:

\section{The clinical viva}

This lasts 50 minutes and consists of a long case and three short cases or scenarios. During the first 10 minutes, you will have the opportunity to view clinical information related to the long case consisting of history, examination findings and investigations, e.g. ECG, chest X-ray, pulmonary function tests and blood results. This is followed by 20 minutes of questioning related to this case. During the final 20 minutes, the examiners will question you on three further unrelated topics.

\section{The clinical science viva}

This is a 30-minute viva consisting of 4 questions on applied pharmacology, anatomy, physiology and physics. This viva is not within the scope of this book.

\section{An approach to revision for the clinical vivas}

The period between the written paper and viva examination is a stressful time. For the first 2 weeks you do not even know if you have a viva. This makes it difficult to find the motivation to carry on working until the results are posted. The last thing you want to do is continue the cold, factual learning that has made your life such a misery over the last few weeks. You want to go to the pub instead! However, if you are 'invited' to attend for the vivas, you will find yourself wishing you had worked solidly for the 2 weeks since the written paper! What is needed is a change of tack in order to sustain the flagging momentum. We found sitting around in armchairs (cups of tea in hand) discussing anaesthetic topics far preferable to the 'textbook and solitary desk-lamp at midnight' scenario.

It is important to realise that the viva requires a different approach to revision. This book aims to give you a strategy for viva revision that will hopefully make it less tedious.

We initially found our viva technique left much to be desired, despite adequate knowledge. There is a particular 'knack' to passing this type of 
Cambridge University Press

978-0-521-72018-2 - The Clinical Anaesthesia Viva Book: Second edition

Julian M. Barker, Simon L. Maguire and Simon J. Mills

Excerpt

More information

examination, and possessing a well-honed technique can enable you to appear confident and knowledgeable (even if you don't feel it!). With the experience you have gained as a trainee, your knowledge base is very likely to be good enough to tackle most questions. However, it is the way in which you communicate your knowledge that will need to impress the examiners. As in all branches of anaesthesia, there are many ways to skin a cat, and revising for the clinical viva is no exception. We found the following techniques extremely valuable in the run-up to the vivas:

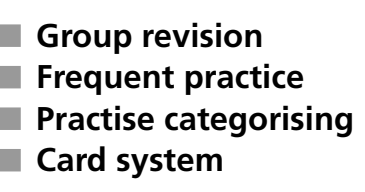

\section{Group revision}

It is extremely useful to team up with some friends or colleagues regularly in the weeks before the viva and practise talking about anaesthetic topics.

Practising with friends has several advantages:

- The whole exam period is very stressful and seeing your friends on a regular basis will help keep you sane. This is better than locking yourself in a small room with a pile of books and trying to learn the coagulation cascade for the fifth time since qualification!

- Your morale will remain in better shape than if you were revising on your own because you will be able to encourage each other. You will also be more aware of the progress you are making.

- As a group, you can pool your resources in terms of reference books and previous questions. During the working day, one of you may have had a practice viva with a consultant who asked an awkward question or a common question asked in a different way. You can then discuss with your friends how they would have answered it.

Different people revise in different ways and, consequently, will have their own way of talking about a subject. This means that others in the group will benefit from listening to the practice viva. They may have a particular piece of knowledge that really helps an answer gel together or they may use a particular turn-of-phrase that succinctly deals with a potential minefield.

- You can practise phrasing your answers in a particular way in the knowledge that, if it all falls apart halfway through, it won't matter and you can have another go. This is less easy to do in front of consultants who might write your reference!

By being 'the examiner', you will gain insight into the pitfalls of the viva process. You can usually see someone digging a hole for themselves a mile off!

\section{Frequent practice}

\section{Repetition of clinical scenarios}

During your revision, you will find the same clinical situations coming up time and time again (as in the exam). Over the years, anaesthetic techniques may 
Cambridge University Press

978-0-521-72018-2 - The Clinical Anaesthesia Viva Book: Second edition

Julian M. Barker, Simon L. Maguire and Simon J. Mills

Excerpt

More information

change but new techniques are all aimed at trying to solve particular clinical problems, for example, the fibre-optic scope to help with the difficult airway or new drugs that provide more cardiovascular stability. However, the problems remain the same! Patients will still present with difficult airways, ischaemic heart disease, COAD, obesity, hypertension, etc. The more you practise, the more often you will find yourself repeating the problems each of these scenarios presents and thus the more confident and slick you will become at delivering the salient points.

There are obviously a few exceptions, e.g. MRI scanners and laser surgery, where the advancement of technology has presented new challenges to the anaesthetist. These situations are in the minority and as long as you are aware of them and the associated anaesthetic problems, you should be well-equipped to deal with questions on them in the exam.

The clinical scenarios break down into a few categories:

Medical conditions that have anaesthetic implications, e.g.

Aortic stenosis

Diabetes

Hyperthyroidism.

Surgical procedures that have anaesthetic implications, e.g.

Oesophagectomy

CABG

Pneumonectomy.

Anaesthetic emergencies/difficult situations, e.g.

Anaphylaxis

Malignant hyperthermia

Failed intubation.

- Paediatric cases. These represent a limited range of cases the examiners are likely to ask you about, e.g.

Upper airway obstruction

Pyloric stenosis

Bleeding tonsil.

Having repeatedly practised these clinical scenarios, you will soon realise that the problems of anaesthetising an obese patient with diabetes, ischaemic heart disease, porphyria and myasthenia for an abdominal aortic aneurysm repair (!) can be broken down into the problems that the respective conditions present to the anaesthetist, plus the problems of the specific operation. You may then approach what seems to be a nightmare question with a degree of confidence and structure.

\section{Phrasing}

It cannot be over-emphasized that frequent practice will improve your viva technique. As already mentioned, some topics crop up again and again in different situations, such as part of a long case or even a complete short case (e.g. obesity, anaesthesia for the elderly or the difficult airway). With regular practice, you will soon develop your own 'patter' to help you deal with these common clinical scenarios. These can then be adopted at opportune moments to buy yourself easy marks whilst actually giving you time to gather your thoughts. 
Cambridge University Press

978-0-521-72018-2 - The Clinical Anaesthesia Viva Book: Second edition

Julian M. Barker, Simon L. Maguire and Simon J. Mills

Excerpt

More information

\section{Practise categorising}

Putting order to your answers demonstrates to the examiners that you conduct your clinical practice in a systematic and safe way. If you do not mention the most important points first (e.g. airway problems in a patient presenting with a goitre), then this may suggest to the examiners that you are disorganized. An 'ABC' (order of priority) approach to many of the questions may be helpful. For example, in obese patients, managing the airway has a higher priority than difficulty with cannulation.

It is often a good idea to use your opening sentence to tell the examiners how you are going to categorize your answer.

\section{Example 1:}

'Tell me about the anaesthetic implications of rheumatoid arthritis'.

'Patients with rheumatoid arthritis may have a difficult airway and secondary respiratory and cardiovascular pathology. They are frequently anaemic, taking immunosuppressant drugs and the severe joint pathology leads to problems with positioning'.

\section{Example 2:}

'What are the important considerations when anaesthetising a patient for a pneumonectomy'?

'These may be divided into three broad areas: the pre-operative assessment of fitness for pneumonectomy and optimisation, the conduct of anaesthesia with particular reference to one-lung anaesthesia, positioning, intra-operative monitoring and fluid balance and finally post-operative care'.

\section{Card system}

We formatted postcards to summarise the main problems associated with different anaesthetic situations. These proved to be a good starting point for viva practice and a quick source of reference. They also encouraged us to deliver the first few points in a punchy manner.

\section{For example:}

'What problems do you anticipate with anaesthetising a patient with Down's syndrome'?

'These patients present the following problems for the anaesthetist. They may have a difficult airway, an unstable neck, cardiac abnormalities, mental retardation, epilepsy and a high incidence of hepatitis B infection'.

\section{Viva technique}

\section{Think first}

The opening sentence 
Cambridge University Press

978-0-521-72018-2 - The Clinical Anaesthesia Viva Book: Second edition

Julian M. Barker, Simon L. Maguire and Simon J. Mills

Excerpt

More information

\section{Categorise or die!}

The long case

\section{Think first}

Don't panic. If you are unlucky enough to be asked a question about an obscure subject such as lithium therapy (as two of us were in our science viva), remember the examiners have only just seen the questions as well. It may also be of some comfort to know that there will be at least ten other candidates being asked the same question at the same time. Keep things simple at first and think about how you are going to structure your answer. Categorising your answer may allow you to deliver more information about the topic than you thought you knew. Conversely, do not dwell on what you do not know, e.g. the $\mathrm{pH}$ and dose!

\section{Example: 'Tell me about lithium'}

Think... 'What is it used for'?

Say... 'Lithium is a drug used in the treatment of mania and the prophylaxis of manic depression'.

Think... 'What is the presentation and dose?... I don't know the dose'.

Say... 'It is presented in tablet form'.

Think... 'What is its mode of action? ... I have no idea but I know it is an antipsychotic'!

Say... 'Its main action is as an antipsychotic'.

Think... 'Why are they asking me this question? What is the relevance to anaesthetic practice'?

Say... 'It has a narrow therapeutic range and therefore toxicity must be looked for. Side effects may include nausea, vomiting, convulsions, arrhythmias and diabetes insipidus with hypernatraemia'.

A similar approach can be used for the clinical viva.

\section{The opening sentence}

This will set the tone of the viva. If the first words to come from your mouth are poorly structured, ill thought-out or just plain rubbish, then you are likely to annoy the examiners and will face an uphill struggle. If, on the other hand, your first sentence is coherent, succinct and structured, then you will be half-way there. With a bit of luck, the examiners will sit back, breathe a sigh of relief (because it has been a very long day for them) and allow you to demonstrate your obvious knowledge of the subject in hand!

\section{For example:}

'What are the problems associated with anaesthesia for thyroid disease'?

'Anaesthesia for patients with thyroid disease has implications in the pre-, intra- and post-operative periods'. 
Cambridge University Press

978-0-521-72018-2 - The Clinical Anaesthesia Viva Book: Second edition

Julian M. Barker, Simon L. Maguire and Simon J. Mills

Excerpt

More information

You are then able to expand in a logical way from here.

'Pre-operatively, assessment of the airway and control of the functional activity of the gland is essential...'

\section{Categorise or die!}

Remember this lends structure to your answer and gives the examiners the impression you are about to talk about the subject with authority. If you categorise your answer well enough, they may actually stop you and move onto something else.

\section{The long case}

The above points relate to the short and long cases but there are aspects of the long case that can be anticipated. Some answers can therefore be prepared in advance.

\section{Opening question}

You will be asked to summarise the case so prepare your opening sentence beforehand.

\section{For example:}

You may be asked to summarise the scenario of a 75-year-old man with chronic obstructive pulmonary disease who is scheduled to undergo an elective cardio-oesophagectomy the following day.

'Would you like to summarise the case'?

One possible answer may begin:

'This is an elderly gentleman with complex medical problems who is scheduled for a cardio-oesophagectomy. He has evidence of chronic obstructive pulmonary disease, ischaemic heart disease and diabetes. There will be substantial strain on his cardio-respiratory system. This operation is a major procedure that involves considerable fluid shifts, a potential for large blood loss and requires careful attention to analgesia. These are the main issues that I would concentrate on in my pre-operative assessment'.

Even though a cardio-oesophagectomy involves other considerations (e.g. double-lumen tube / one-lung ventilation) it can be seen that this opening sentence could be adapted to suit other clinical scenarios such as:

Pneumonectomy

Laparotomy

CABG / valve replacement

Cystectomy

Open prostatectomy 
Cambridge University Press

978-0-521-72018-2 - The Clinical Anaesthesia Viva Book: Second edition

Julian M. Barker, Simon L. Maguire and Simon J. Mills

Excerpt

More information

\section{Analyse all the investigations}

You will be asked for your opinion on the ECG, chest X-ray, blood results, etc., so make sure you have decided on the abnormalities and the most likely causes for them in the 10 minutes you have to view the data. Try to make your answers punchy and authoritative.

For example, 'The ECG shows sinus rhythm with a rate of 80 and an old inferior infarct' is better than going through the ECG in a painstaking 'The rate is ... the rhythm is... the axis is...'

Don't waste valuable time waffling on about the normal-looking bones on a chest X-ray if there is a barn-door left lower lobe collapse. This does not necessarily imply you are not thorough, providing you demonstrate that you have looked for and excluded other abnormalities.

\section{Anaesthetic technique}

You will usually be asked how you would anaesthetise the patient in the long case. There will often not be a right or wrong answer, but you should try to decide on your technique and be able to justify it. The examiners may only be looking for the principles of anaesthesia for a particular condition such as aortic stenosis, although this is probably more likely in the short cases.

\section{For example:}

'You are asked to provide an anaesthetic for a 77-year-old lady who needs a hemi-arthroplasty for a fractured neck of femur. She had a myocardial infarction 3 months ago and has evidence of heart failure'.

You should be able to summarise the principles involved and choose an anaesthetic technique appropriate to the problems presented. You could, for example, give this patient a general anaesthetic with invasive monitoring (PAFC, A-line, etc.), you could use TIVA with remifentanil or a neuroaxial block. All of these techniques could be justified, but to simply say that you would use propofol, fentanyl and a laryngeal mask without saying why, may be asking for trouble!

In some circumstances it may be the options for management rather than a specific technique that is required. You may find it appropriate to list the options for analgesia in a patient having a pneumonectomy, for example, and then say why you would use one technique over the others.

You should try to address the anaesthetic technique for the long case BEFORE you face the examiners. You will not look very credible if you have had 10 minutes to decide on this and have not reached some kind of conclusion.

Overall, most candidates felt that the examiners were pleasant and generally helpful. If you are getting sidetracked they will probably give you a hint so you do not waste time talking about something for which there are no allocated marks. If they do give you a hint, take it! Good luck. 


\title{
The Short Cases
}

\section{Abdominal aortic aneurysm rupture}

You are called to the ward to see a 74-year-old man with a ruptured aortic aneurysm. His blood pressure is 70/40.

What are the major problems in managing a ruptured $A A A$ ?

\author{
Pre-operatively \\ - Severe hypovolaemia \\ - Initial fluid resuscitation must be cautious \\ - Assessment of concomitant medical problems \\ - Patients are usually 'arteriopaths' with significant coronary disease \\ No time for lengthy investigations \\ - Access to vascular surgery - may need to transfer out
}

\section{Intra-operatively}

- Cardiovascular instability Induction

Before aortic cross-clamping

When the clamp is removed

Large blood losses Blood, FFP and platelets required

Effects of massive transfusion

Temperature control

Metabolic acidosis

\section{Post-operatively}

Respiratory support may be required for poor gas exchange and metabolic acidosis.

- Cardiovascular complications include haemorrhage, myocardial and lower limb ischaemia.

- Renal failure is common due to peri-operative hypotension, aortic cross-clamping (infra-renal clamp still significantly reduces renal blood flow by about $40 \%$ ), atheromatous emboli, surgical insult, intra-abdominal hypertension ( $>12 \mathrm{mmHg}$ ) or compartment syndrome $(>20 \mathrm{mmHg})$. 
Cambridge University Press

978-0-521-72018-2 - The Clinical Anaesthesia Viva Book: Second edition

Julian M. Barker, Simon L. Maguire and Simon J. Mills

Excerpt

More information

- Neurological sequelae such as paraplegia or stroke may occur secondary to damaged spinal arteries or embolic/ischaemic events.

\section{What is your immediate management on the ward?}

ABC approach - highest $\mathrm{FiO}_{2}$ obtainable should be commenced.

Two large-bore intravenous cannulae should be inserted and fluids given.

\section{How much fluid would you use?}

This would depend on the blood pressure and the clinical state of the patient. A patient who has an unrecordable blood pressure and is about to arrest should be given fluids quickly, but in this man fluids should be given cautiously. Repeated $250 \mathrm{ml}$ fluid boluses titrated to physiological endpoints (consciousness, base deficit, lactate) should be used. One should not necessarily aim to restore blood pressure to 'normal' as this may reverse vasoconstriction and disrupt fibrin clots that were contributing to haemostasis.

\section{What else would you do?}

Take blood for full blood count, urea and electrolytes, clotting screen, blood gas.

Cross-match for 10 units, consider type O-negative or group-specific blood.

Second anaesthetist (preferably consultant) is required.

- Haematology should be alerted to the need for large volumes of blood, FFP and platelets.

- An assessment of co-existing medical problems and the likelihood of difficult intubation should be made.

Do not delay surgery whilst awaiting lengthy investigations.

Transfer the patient to the operating theatre as soon as possible.

Only haemodynamically stable patients can be taken for CT scanning to diagnose rupture and assess suitability for open or endovascular repair.

\section{What monitoring would you use?}

ECG, non-invasive $\mathrm{BP}, \mathrm{SpO}_{2}$ and capnography initially.

- Surgery should not be delayed by prolonged attempts to insert arterial and central lines at this stage.

\section{How would you proceed with anaesthesia?}

\section{Big drips}

All vaso-active drugs should be drawn up prior to induction.

Blood should be immediately available. 
Cambridge University Press

978-0-521-72018-2 - The Clinical Anaesthesia Viva Book: Second edition

Julian M. Barker, Simon L. Maguire and Simon J. Mills

Excerpt

More information

A method of delivering warmed fluids rapidly and continuously is beneficial such as a 'Level- $1^{\text {TM }}$ infusor'.

Anaesthetise in theatre on the table

A rapid sequence induction is performed with the surgeon scrubbed and the patient already cleaned and draped (muscle relaxation may release the tamponade on the aorta worsening bleeding and the combined effects of induction agents and IPPV can cause profound hypotension).

- Anaesthesia is maintained with an appropriate agent in oxygen/air.

- Avoid nitrous oxide because bowel distension may increase intra-abdominal pressure post-operatively.

When the cross-clamp is on and there is 'relative' stability, invasive lines may be inserted if not already in place.

- Temperature probe

Nasogastric tube

- Urinary catheter

- Active warming such as with a warm air blower over the chest helps to maintain temperature, but should be avoided on the legs during clamping.

Loop diuretics (e.g. furosemide), dopamine, mannitol, fenoldapam and $\mathrm{N}$-acetylcysteine have been proposed as renoprotective agents. There is no Level 1 evidence to support their use. The mainstay of renal preservation is maintenance of renal oxygen delivery and the avoidance of nephrotoxins (e.g. non-steroidal anti-inflammatory drugs, angiotensin-converting enzyme inhibitors, contrast and aminoglycosides).

\section{How would you control the hypertension associated with cross-clamping?}

SVR may rise by up to $40 \%$ resulting in myocardial ischaemia. If increasing the inspired volatile concentration and giving opioid and/or propofol are not effective, then GTN can be used, especially if myocardial ischaemia is present.

\section{How would you manage the patient at the end of the operation?}

- Intensive care is usually required.

- Sedation and ventilation may need to be continued until the temperature is corrected, cardiovascular stability is established and acid/base status and gas exchange are acceptable.

- Predictors of survival to discharge include patient age, total blood loss and post-operative hypotension.

\section{Bibliography}

Cowlishaw P, Telford R. (2007). Anaesthesia for abdominal vascular surgery. Anaesthesia and Intensive Care Medicine, 8(6), 248-52.

Leonard A, Thompson J. (2008). Anaesthesia for ruptured abdominal aortic aneurysm. Continuing Education in Anaesthesia, Critical Care and Pain, 8(1), 11-15.

Sakalihasan N, Limet, R, Defawe O. (2005). Abdominal aortic aneurysm. Lancet, 365, 1577-89. 